\title{
Phase transition in a system of 1D harmonic oscillators obeying Polychronakos statistics with a complex parameter
}

\author{
Andrij Rovenchak \\ Department for Theoretical Physics, Ivan Franko National University of Lviv \\ 12 Drahomanov Str., Lviv, UA-79005, Ukraine \\ E-mail: andrij.rovenchak@gmail.com
}

Received February 25, 2013, revised March 28, 2013

\begin{abstract}
PACS: $05.30 . P r \quad$ Fractional statistics systems (anyons, etc.);
67.10.Fj Quantum statistical theory;

64.60.-i General studies of phase transitions.
\end{abstract}

For a system of 1D harmonic oscillators obeying Polychronakos statistics with a complex parameters the emergence of a phase transition is reported and temperature dependences of energy and heat capacity are studied in detail. Estimations towards a possibility to check the obtained jumps in the specific heat are made.

Keywords: Polychronakos statistics, phase transitions.

\section{Introduction}

In recent decades, quantum systems obeying fractional statistics have been a subject of studies based on different approaches [1]. In real physical systems a description based on an intermediate statistics between the Fermi and Bose cases appears in a number of possibilities: the socalled exclusion statistics can be linked to the interaction in 1D systems [2]; ultracold gases can be described by means of anyon-fermion mapping [3]; thermodynamics of finite Bose-systems can be studied using the Gentile statistics [4]; and this list can be expanded further.

In this work, a generalization of the Polychronakos statistics [5-7] is studied. The parameter of statistics is considered a complex number [8]. Complex-valued physical quantities can occur effectively in some physical systems: complex energy is connected to a dissipative process [9]; complex chemical potential is also used, e.g., in quantum chromodynamics [10] or physics of semiconductors [11]; complex external potential is applied to describe the interaction between the light field and atoms moving in crystals $[12,13]$ and also can be used to study PT-symmetry breaking [14].

The one-dimensional problem is considered here for the sake of simplicity. A system of 1D harmonic oscillators effectively corresponds to a 2D homogenous system, which opens a possibility to apply the obtained results for studies of, e.g., critical phenomena in liquid-helium films [15-17] or surface-electron systems [18].

The paper is organized as follows. In Sec. 2 statistics is explained and calculation procedure is described. In Sec. 3 analytical expressions for the thermodynamic functions of a 1D harmonic oscillator system are derived. Main results are obtained in Sec. 4, where the critical temperature is calculated and temperature dependence of energy and heat capacity is analyzed. A discussion on possible experimental observation of the phase transition is given in Sec. 5 .

\section{Initial expressions}

In the Polychronakos statistics [5,6] the occupation of the $j$ th level with energy $\varepsilon_{j}$ is given by

$$
n_{j}=\frac{1}{z^{-1} e^{\varepsilon_{j} / T}-\alpha},
$$

where $z$ is fugacity and $T$ is temperature. Here $n_{j}$ is a formal characteristic and can take a complex values. Note that this form for the occupation numbers was also suggested a priori by Acharya and Narayana Swamy to describe anyons [19].

To make a generalization, the parameter $\alpha=\mathrm{e}^{i \pi v}=$ $=\alpha^{\prime}+i \alpha^{\prime \prime}$ is put a complex number; $v=0-1$ provides a smooth transition between the bosonic and fermionic 


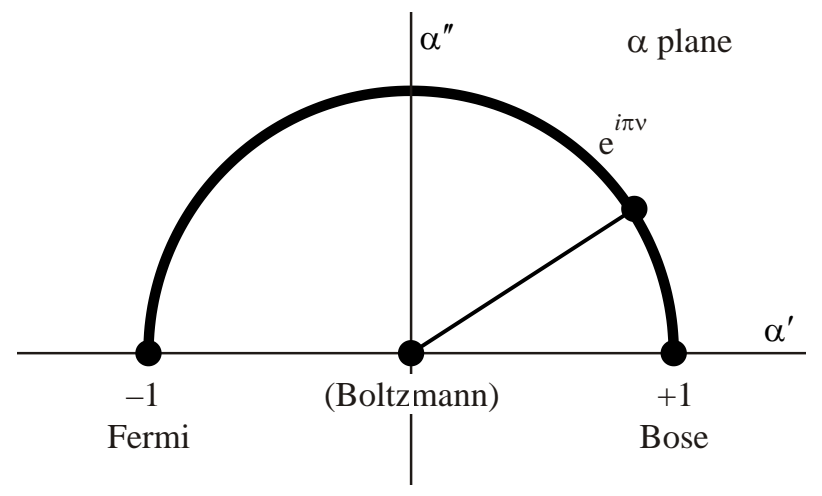

Fig. 1. Statistics explanation.

limits, while the value $\alpha=0$ corresponding to the classical Boltzmann statistics is avoided, see Fig. 1 [8].

It can be shown that in the limit $v \rightarrow 0$ a simple correspondence can be established between a system with the real excitation spectrum $\varepsilon_{j}$ obeying the Polychronakos statistics generalized as above and a system with a small dissipative term in the excitation spectrum obeying the Bose statistics. If the elementary excitation spectrum is $\varepsilon_{j}+i \gamma_{j}$, where $\gamma_{j}$ corresponds to small energy dissipation, for high levels with $j \gg 1$ the link is $\gamma_{j}=\pi v T \varepsilon_{j}$ [8]. Such systems are seen as most prospective candidates for experimental observations of the effects discussed in the present work.

Calculations are made using a simple scheme as follows.

The number of particles $N$ is given by

$$
N=\sum_{j} g_{j} n_{j}=\sum_{j} \frac{g_{j}}{z^{-1} \mathrm{e}^{\varepsilon_{j} / T}-\alpha},
$$

where $g_{j}$ is the degeneration of the $j$ th level. Elementary excitation spectrum $\varepsilon_{j}$ is assumed real. Since $N$ is a real number, fugacity $z=z^{\prime}+i z^{\prime \prime}$ must be a complex number.

Energy is then given by

$$
E=\sum_{j} \varepsilon_{j} g_{j} n_{j}=\sum_{j} \frac{\varepsilon_{j} g_{j}}{z^{-1} \mathrm{e}^{\varepsilon_{j} / T}-\alpha}=\mathcal{E}+i \Gamma
$$

with $z=z(N, T)$ defined from Eq. (2). Note that only such values of the statistics parameter $v$ and other quantities are physically reasonable which correspond to $\Gamma \ll \mathcal{E}$; otherwise the system becomes essentially nonequilibrium making the whole subsequent consideration incorrect.

Real and imaginary parts of the heat capacity equal

$$
C=\frac{d \mathcal{E}}{d T}, \quad \Theta=\frac{d \Gamma}{d T} .
$$

The peculiarities of the temperature behavior are better seen on the specific heat curves, and these will be given a closer look further.

\section{1D oscillators}

Let us consider a system of 1D harmonic oscillators with frequency $\omega$. The degeneracy of the $j$ th level equals unity, $g_{j}=1$. The excitation spectrum is

$$
\varepsilon_{j}=\hbar \omega j, \quad j=0,1,2,3, \ldots
$$

The quantity $\hbar \omega$ is also used as a unit of temperature.

One-dimensional systems of oscillators can be realized in highly anisotropic harmonic traps [20] or in optical lattices [21,22].

To obtain results in an analytic form, the summation in Eqs. (2), (3) is substituted with integration using the density of state function $g(\varepsilon): \sum_{j} \rightarrow \int d \varepsilon g(\varepsilon)$. The accuracy of this approximation increases for the number of particles being large and if temperature is large comparing to the level separation, $T \gg \hbar \omega$. Numerical comparison of the results obtained using exact summation and integration shows a very good agreement already for the number of particles $N=1000$.

For a system of 1D harmonic oscillators $g(\varepsilon)=$ const, which makes it equivalent to, e.g., a system of free particles trapped to a 1D harmonic potential (in the semiclassical approach [23]) or a 2D system of free particles in a box.

In the case under consideration, $g(\varepsilon)=1 / \hbar \omega$. The number of particles is then

$$
N=\frac{1}{\hbar \omega} \int_{0}^{\infty} \frac{d \varepsilon}{z^{-1} \mathrm{e}^{\varepsilon / T}-\alpha}=-\frac{T}{\hbar \omega \alpha} \ln (1-z \alpha)
$$

giving fugacity as

$$
z=\frac{1}{\alpha}\left(1-\mathrm{e}^{-\hbar \omega N \alpha / T}\right) .
$$

The energy equals

$$
\begin{gathered}
E=\frac{1}{\hbar \omega} \int_{0}^{\infty} \frac{\varepsilon d \varepsilon}{z^{-1} \mathrm{e}^{\varepsilon / T}-\alpha}=\frac{T^{2}}{\hbar \omega \alpha} \operatorname{Li}_{2}(z \alpha)= \\
=\frac{T^{2}}{\hbar \omega \alpha} \operatorname{Li}_{2}\left(1-\mathrm{e}^{-\hbar \omega N \alpha / T}\right),
\end{gathered}
$$

where $\operatorname{Li}_{s}(x)$ is the polylogarithm:

$$
\operatorname{Li}_{s}(x)=\sum_{k=1}^{\infty} \frac{x^{k}}{k^{s}} .
$$

This series definition is valid for all complex $s$ and complex $x$ with $|x|<1$; for other values of $x$ an analytic continuation is used.

Using the obvious property

$$
\frac{d \mathrm{Li}_{s}(x)}{d x}=\frac{1}{x} \operatorname{Li}_{s-1}(x)
$$


and taking into account that $\operatorname{Li}_{1}(x)=-\ln (1-x)$, for the heat capacity one obtains

$$
C+i \Theta=\frac{d E}{d T}=N \frac{\hbar \omega N \alpha / T}{1-\mathrm{e}^{\hbar \omega N \alpha / T}}+N \frac{2 T}{\hbar \omega N \alpha} \operatorname{Li} 2\left(1-\mathrm{e}^{-\hbar \omega N \alpha / T}\right) .
$$

As one should expect for extensive quantities, $E \propto N$ and $C \propto N$, which is not immediately seen from the above expressions (8) and (10). To ensure extensive nature of energy and heat capacity, thermodynamic limit must be taken into consideration, which in the case of 1D harmonic oscillators reads $\omega N=$ const. Such a result can be obtained in a number of different ways [4,20], cf. also [24]. The easiest interpretation of such a thermodynamic limit can be given as follows. If a $D$-dimensional system is contained in a harmonic potential with frequency $\omega$, classical turning points confine the particles to some characteristic length $r \propto 1 / \omega$. To keep an average density $N / r^{D}$ constant, the requirement $N \omega^{D}=$ const must be satisfied.

Thus, in the case under consideration

$$
\frac{T}{\hbar \omega}=\frac{N T}{\hbar \omega N}=\frac{N T}{\text { const }} \text { and } \quad \frac{\hbar \omega N}{T}=\frac{\text { const }}{T},
$$

therefore, the conditions $E \propto N$ and $C \propto N$ hold.

\section{Critical temperature and behavior of thermodynamic functions}

Discontinuities are observed in both energy and heat capacity as functions of temperature. These discontinuities are due to $\mathrm{Li}_{2}(z \alpha)$, where the argument is changing the sign of the imaginary part.

Indeed, in the calculation of the integral one obtains singularity " $1 / 0$ " when

$$
\begin{gathered}
\mathrm{e}^{\varepsilon / T}-\mathrm{z} \alpha=0=\mathrm{e}^{\varepsilon / T}-1+\mathrm{e}^{-\hbar \omega N \alpha / T}= \\
=\mathrm{e}^{\varepsilon / T}-1+\mathrm{e}^{-\frac{\hbar \omega N}{T} \alpha^{\prime}}\left(\cos \frac{\hbar \omega N}{T} \alpha^{\prime \prime}-i \sin \frac{\hbar \omega N}{T} \alpha^{\prime \prime}\right)
\end{gathered}
$$

if

$$
\frac{\hbar \omega N}{T} \alpha^{\prime \prime}=\pi n \quad \text { and } \quad \cos \pi n=(-1)^{n}<0
$$

A set of critical temperature values can thus be defined as follows:

$$
T_{C}^{(k)}=\hbar \omega N \frac{\alpha^{\prime \prime}}{(2 k+1) \pi}, \quad k=0,1,2,3, \ldots,
$$

cf. Fig. 2. For brevity, the index “( $k$ )" will be dropped in further derivations.

To estimate the contribution from the singularity into thermodynamic function, the following procedure can be applied. Let the singularity corresponds to $\varepsilon_{0}$ such that $\mathrm{e}^{\varepsilon_{0} / T}=\mathrm{z} \alpha$. The energy $E$ can be expressed as follows:

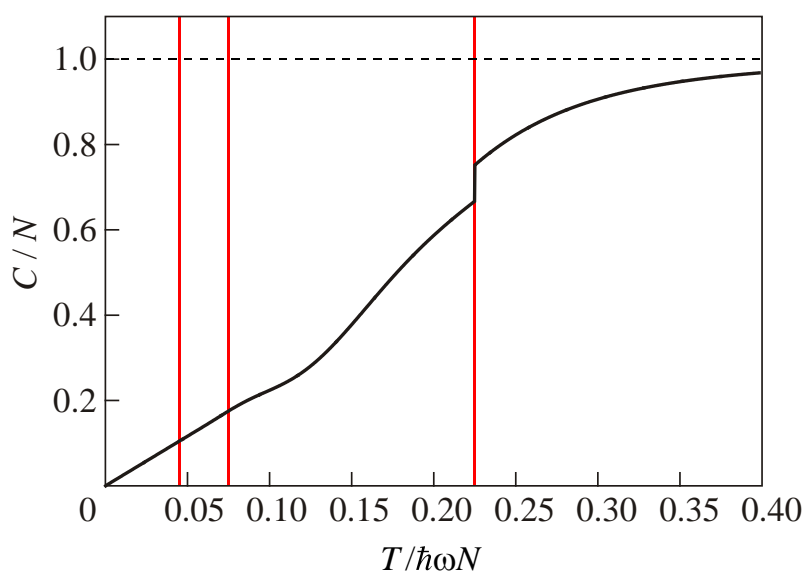

Fig. 2. Real part of the specific heat $C / N$ at $v=0.25$. Vertical lines show critical temperatures. The right-most one corresponds to $k=0$.

$$
\begin{gathered}
E=\frac{z}{\hbar \omega} \int_{0}^{\infty} \frac{\varepsilon d \varepsilon}{\mathrm{e}^{\varepsilon / T}-z \alpha}=\frac{z}{\hbar \omega} \int_{0}^{\varepsilon_{0}-\Delta} \frac{\varepsilon d \varepsilon}{\mathrm{e}^{\varepsilon / T}-z \alpha}+ \\
+\frac{z}{\hbar \omega} \int_{\varepsilon_{0}+\Delta}^{\infty} \frac{\varepsilon d \varepsilon}{\mathrm{e}^{\varepsilon / T}-z \alpha}+\frac{z}{\hbar \omega} \int_{\varepsilon_{0}-\Delta}^{\varepsilon_{0}+\Delta} \frac{\varepsilon d \varepsilon}{\mathrm{e}^{\varepsilon / T}-\mathrm{e}^{\varepsilon_{0} / T}},
\end{gathered}
$$

where $\Delta$ is a small positive number. In the limit of $\Delta \rightarrow 0$ first two terms give a smooth function of temperature $E_{\text {smooth }}$, and the last term can be written only to the first order of $\left(\varepsilon-\varepsilon_{0}\right)$ :

$$
E=E_{\text {smooth }}(T)+\frac{z T}{\hbar \omega} \mathrm{e}^{-\varepsilon_{0} / T} \int_{\varepsilon_{0}-\Delta}^{\varepsilon_{0}+\Delta} \frac{\varepsilon d \varepsilon}{\varepsilon-\varepsilon_{0}} .
$$

While $\varepsilon_{0}$ is real for $T=T_{C}$, it becomes complex as soon as $T$ deviates from the critical point. The sign of the imaginary part is defined by $\sin \left(\hbar \omega N \alpha^{\prime \prime} / T\right)$, see (11). Therefore, the denominator $\left(\varepsilon-\varepsilon_{0}\right)$ must be substituted with $\left(\varepsilon-\varepsilon_{0} \mp i \eta\right)$ for $T=T_{C} \pm 0$, where $\eta \rightarrow+0$. Using Sochocki's formulas [25], one can write

$$
\frac{1}{\varepsilon-\varepsilon_{0} \mp i \eta}=v \cdot p \cdot \frac{1}{\varepsilon-\varepsilon_{0}} \pm i \pi \delta\left(\varepsilon-\varepsilon_{0}\right),
$$

where "v.p." denotes principal value and $\delta\left(\varepsilon-\varepsilon_{0}\right)$ is Dirac's delta-function.

Thus, in the vicinity of critical points energy equals

$$
E\left(T_{C} \pm 0\right)=E_{\text {smooth }}\left(T_{C}\right) \pm i \pi \frac{T_{C}}{\hbar \omega} z\left(T_{C}\right) \varepsilon_{0} \mathrm{e}^{-\varepsilon_{0} / T_{C}} .
$$

For the real part one obtains

$$
\Delta \mathcal{E}\left(T_{C}\right)=\mathcal{E}\left(T_{C}+0\right)-\mathcal{E}\left(T_{C}-0\right)=-2 \pi \frac{T_{C}}{\hbar \omega} z^{\prime \prime}\left(T_{C}\right) \varepsilon_{0} \mathrm{e}^{-\varepsilon_{0} / T_{C}} .
$$

Taking into account the definition of critical temperature (12) and writing the statistics parameter in the form 
$\alpha=\cos \pi \nu+i \sin \pi \nu$, after simple transformations the following results can be obtained for jumps of the real parts of energy and heat capacity:

$$
\begin{gathered}
\frac{1}{N} \frac{\Delta \mathcal{E}\left(T_{C}\right)}{\hbar \omega N}=\frac{2 \sin ^{3} \pi \nu}{(2 k+1)^{2} \pi} \ln \left(1+\mathrm{e}^{-(2 k+1) \pi \cot \pi \nu}\right), \\
\frac{1}{N} \Delta C\left(T_{C}\right)=\frac{4 \sin ^{2} \pi v}{(2 k+1)} \ln \left(1+\mathrm{e}^{-(2 k+1) \pi \cot \pi v}\right) .
\end{gathered}
$$

In the bosonic limit $v \rightarrow 0$ :

$$
\begin{aligned}
& \frac{1}{N} \frac{\Delta \mathcal{E}\left(T_{C}\right)}{\hbar \omega N}=\frac{2 \pi^{2} v^{3}}{(2 k+1)^{2}} \mathrm{e}^{-(2 k+1) / v}, \\
& \frac{1}{N} \Delta C\left(T_{C}\right)=\frac{4 \pi^{2} v^{2}}{(2 k+1)} \mathrm{e}^{-(2 k+1) / v} .
\end{aligned}
$$

The jumps are finite as soon as $v>0$, but the most pronounced one corresponds to $k=0$ and then they rapidly vanish for $k>0$, see Table 1 . Note that for values $v \gtrsim 0.35$ the oscillations of $C(T)$ in the low-temperature domain increase and lead to some unphysical regions with negative specific heat.

Table 1. Jumps of the specific heat $C / N$ at the critical temperature

\begin{tabular}{c|c|c|c}
\hline \hline$v$ & $k=0$ & $k=1$ & $k=2$ \\
\hline \hline 0.01 & $1.5 \cdot 10^{-46}$ & $7.5 \cdot 10^{-134}$ & $6.6 \cdot 10^{-221}$ \\
0.05 & $2.4 \cdot 10^{-10}$ & $4.7 \cdot 10^{-28}$ & $1.7 \cdot 10^{-45}$ \\
0.10 & $2.4 \cdot 10^{-5}$ & $3.2 \cdot 10^{-14}$ & $7.7 \cdot 10^{-23}$ \\
0.15 & $1.7 \cdot 10^{-3}$ & $2.6 \cdot 10^{-9}$ & $6.7 \cdot 10^{-15}$ \\
0.20 & $1.8 \cdot 10^{-2}$ & $1.1 \cdot 10^{-6}$ & $1.1 \cdot 10^{-10}$ \\
0.25 & $8.5 \cdot 10^{-2}$ & $5.4 \cdot 10^{-5}$ & $6.0 \cdot 10^{-8}$ \\
0.30 & $2.5 \cdot 10^{-1}$ & $9.3 \cdot 10^{-4}$ & $5.8 \cdot 10^{-6}$ \\
0.34 & $5.0 \cdot 10^{-1}$ & $5.7 \cdot 10^{-3}$ & $1.1 \cdot 10^{-4}$ \\
\hline \hline
\end{tabular}

Notes: While the jump values of $10^{-14}$ and smaller are experimentally unobservable, they are preserved in the Table to show how rapidly $\Delta C$ vanish as $v \rightarrow 0$ and/or $k$ increases.

Low- and high-temperature limits of the heat capacity are easily calculated using series expansions in Eqs. (8) or (10). A linear dependence $C \propto T$ observed in Fig. 3 in the low-temperature domain is

$\frac{C+i \Theta}{N}=\frac{2 T}{\hbar \omega N \alpha} \operatorname{Li}_{2}(1)=\frac{T}{\hbar \omega N} \frac{\pi^{2}}{3}(\cos \pi \nu-i \sin \pi \nu)$,

where the link between the polylogarithm and Riemann's zeta-function is taken into account: $\operatorname{Li}_{2}(1)=\zeta(2)=\pi^{2} / 6$.

Different approaching to the asymptotic value $C / N \rightarrow 1$ (from below at $v<1 / 4$ and from above at $v>1 / 4$ ) is determined by the following limiting behavior of the specific heat:

$$
\frac{C+i \Theta}{N}=1-\frac{\cos 2 \pi \nu}{36}\left(\frac{\hbar \omega N}{T}\right)^{2}-i \frac{\sin 2 \pi \nu}{36}\left(\frac{\hbar \omega N}{T}\right)^{2},
$$
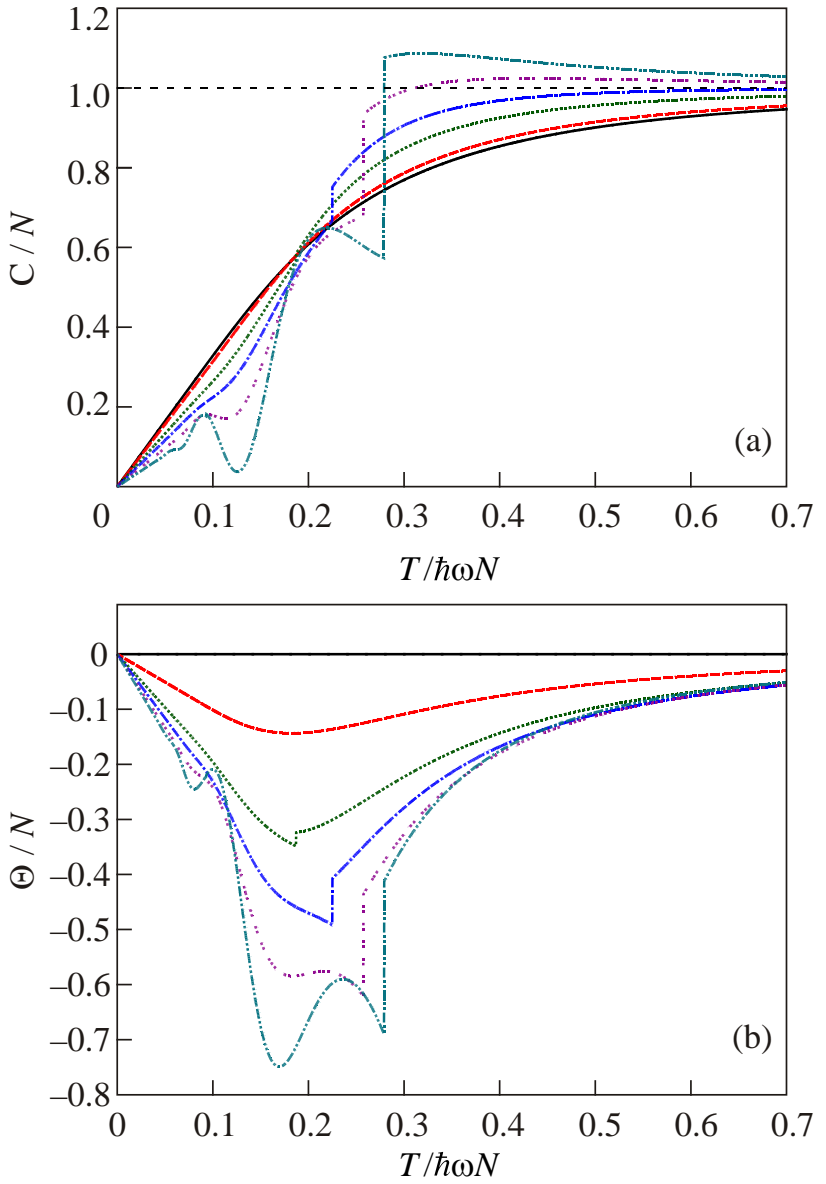

Fig. 3. (Color online) The real (a) and imaginary (b) parts of the specific heat $C / N$ for different values of the parameter $v=0.0$ (black,_—); 0.1 (red, ----); 0.2 (green, ......); 0.25 (blue, .-.-.--); 0.3 (magenta, -- --); 0.34 (cyan, ---.--.--.-.).

where the temperature-dependent term in the real part changes sign at $v=1 / 4$, while the imaginary part remains negative for all $v$, see Fig. 3 .

At $v=1 / 4$ for the real part of the specific heat the leading deviation from the asymptotic value is given by

$$
\frac{C}{N}=1-\frac{1}{1200}\left(\frac{\hbar \omega N}{T}\right)^{4}
$$

\section{Discussion}

Since a discontinuity is found in the energy as a function of temperature, the first-order phase transitions occur at $T_{c}^{(k)}$. It is worth to estimate whether this effect can be tested experimentally.

As it was mentioned in Secs. 1 and 2, complex parameter in the statistics can be obtained as an effective influence of external laser field or dissipation in the elementary excitation spectrum. It is expected, therefore, that the value of $v$ is small, $v \ll 1$. Hence, only the highest critical temperature corresponding to the value $k=0$

$$
T_{C}=\hbar \omega N \frac{\sin \pi \nu}{\pi}
$$


is relevant for the analysis since the jumps of the specific heat become experimentally negligible at $k>1$, see Table 1 .

Recovering the Boltzmann constant $k_{B}$ in order to obtain the value of $T_{C}$ in Kelvins:

$$
k_{B} T_{C}=\hbar \omega N \frac{\sin \pi \nu}{\pi},
$$

one can make simple estimations as follows. Assuming experimental precision in the specific heat measurements as high as $0.01 \%$, which corresponds to $v=0.10-0.15$ (see Table 1), trapping potential frequency $\omega=1 \mathrm{kHz}$, and the number of particles $N=10^{4}$, critical temperature is obtained as $T_{C} \sim 10^{-5} \mathrm{~K}$. The effect thus is available for experimental observations as soon as a proper system, which can be treated within the Polychronakos statistics with a complex parameter, is prepared.

It is interesting to derive from the above results some estimation for a $2 \mathrm{D}$ homogenous system. Its density of states (for spin-0 particles of mass $m$ on the area $S$ ) is easily calculated as $g(\varepsilon)=m S / 2 \pi \hbar^{2}=$ const, which, comparing to the oscillator result $g(\varepsilon)=1 / \hbar \omega$, leads to an effective correspondence:

$$
\omega N=\frac{2 \pi \hbar}{m^{2}} \frac{m N}{S} .
$$

The obtained expression for critical temperature appears to have a structure similar to that for the Kosterlitz-Thouless transition $T_{K T}[26]$ :

$k_{B} T_{C}=\frac{\pi \hbar^{2}}{2 m^{2}} \rho_{2} \frac{2 \sin \pi \nu}{\pi}$ versus $k_{B} T_{K T}=\frac{\pi \hbar^{2}}{2 m^{2}} \rho_{2 s}$,

where the 2D density $\rho_{2}=m N / S$ and $\rho_{2 s}$ is the superfluid density. With a $2 \mathrm{D}$ concentration $\simeq 8 \cdot 10^{18} \mathrm{~m}^{-2}$ corresponding to the bulk liquid helium density of $0.146 \mathrm{~g} / \mathrm{cm}^{3}$ [17], the value of the critical temperature is $T_{C} \lesssim 1 \mathrm{~K}$. Note that this estimation is relevant to an ideal homogenous Bosegas with parameters corresponding to liquid ${ }^{4} \mathrm{He}$. To apply this approach to a realistic planar system, one can proceed from studying phonons - excitations with a linear dispersion - thus a 2D oscillator problem must be analyzed, which is yet to be considered in detail.

\section{Acknowledgments}

I am grateful to Yuri Krynytskyi and Prof. Volodymyr Tkachuk for discussion and useful comments. Remarks from the anonymous referees are highly appreciated.

This work was partly supported by Project ФФ-110Ф (registration No. 0112U001275) from the Ministry of Education and Sciences, Youth and Sports of Ukraine.
1. A. Khare, Fractional Statistics and Quantum Theory, 2nd edition, Singapore, World Scientific (2005).

2. M.T. Batchelor, X.W. Guan, and N. Oelkers, Phys. Rev. Lett. 96, 210402 (2006).

3. M.D. Girardeau, Phys. Rev. Lett. 97, 100402 (2006).

4. A. Rovenchak, Fiz. Nizk. Temp. 35, 510 (2009) [Low Temp. Phys. 35, 400 (2009)].

5. A.P. Polychronakos, Phys. Lett. B 365, 202 (1996).

6. B. Mirza and H. Mohammadzadeh, Phys. Rev. E 82, 031137 (2010).

7. S. Zare, Z. Raissi, H. Mohammadzadeh, and B. Mirza, Eur. Phys. J. C 72, 2152 (2012).

8. A. Rovenchak, J. Phys.: Conf. Ser. 400, 012064 (2012).

9. G.E. Cragg and A.K. Kerman, Phys. Rev. Lett. 94, 190402 (2005).

10. J.R. Ipsen and K. Splittorff, Phys. Rev. D 86, 014508 (2012).

11. P.K. Chakraborty, B. Nag, and K.P. Ghatak, J. Phys. Chem. Solids 64, 2191 (2003).

12. D.O. Chudesnikov and V.P. Yakovlev, Laser Phys. 1, 110 (1991).

13. M.K. Oberthaler, R. Abfalterer, S. Bernet, J. Schmiedmayer, and A. Zeilinger, Phys. Rev. Lett. 77, 4980 (1996).

14. A. Guo, G.J. Salamo, D. Duchesne, R. Morandotti, M. Volatier-Ravat, V. Aimez, G.A. Siviloglou, and D.N. Christodoulides,, Phys. Rev. Lett. 103, 093902 (2009).

15. D.J. Bishop and J.D. Reppy, Phys. Rev. Lett. 40, 1727 (1978).

16. D. Finotello, K.A. Gillis, A. Wong, and M.H.W. Chan, Phys. Rev. Lett. 61, 1954 (1988).

17. V.E. Syvokon, Fiz. Nizk. Temp. 32, 65 (2006) [Low Temp. Phys. 32, 48 (2006)].

18. T.I. Zueva, Yu.Z. Kovdrya, and S.S. Sokolov, Fiz. Nizk. Temp. 33, 3 (2007) [Low Temp. Phys. 33, 1 (2007)].

19. R. Acharya and P. Narayana Swamy, J. Phys. A 27, 7247 (1994).

20. F. Dalfovo, S. Giorgini, and L.P. Pitaevskii, Rev. Mod. Phys. 71, 463 (1999).

21. O. Morsch, J.H. Müller, M. Cristiani, D. Ciampini, and E. Arimondo, Phys. Rev. Lett. 87, 140402 (2001).

22. L. Fallani, L. De Sarlo, J.E. Lye, M. Modugno, R. Saers, C. Fort, and M. Inguscio, Phys. Rev. Lett. 93, 140406 (2004).

23. V. Bagnato and D. Kleppner, Phys. Rev. A 44, 7439 (1991).

24. A. Posazhennikova, Rev. Mod. Phys. 78, 1111 (2006).

25. V.S. Vladimirov, Equations of Mathematical Physics, New York, Marcel Dekker (1971).

26. V.E. Syvokon and K.A. Nasyedkin, Fiz. Nizk. Temp. 38, 8 (2012) [Low Temp. Phys. 38, 6 (2012)]. 